\title{
Gastric antral dysrhythmias in children with chronic idiopathic intestinal pseudoobstruction
}

\author{
S P Devane, A M Ravelli, W M Bisset, V V Smith, B D Lake, P J Milla
}

\begin{abstract}
Chronic idiopathic intestinal pseudoobstruction is a serious disorder of intestinal neuromuscular function resulting in recurrent episodes of intestinal obstruction, and is caused by primary disease of the enteric nerves or enteric smooth muscle. Gastric electrical control activity detected by the non-invasive technique of surface electrogastrography was investigated in 11 children (0.1-16 years) with proven chronic idiopathic intestinal pseudoobstruction (four with known disease of the enteric nerves, three with disease of smooth muscle cells, and four without defined pathology), to determine whether abnormalities were present and whether these were useful in detecting the underlying pathology. Abnormalities were present in eight of 11 patients. Persistent tachygastria (electrical control activity frequency $>5$ cycles/minute) was found in three patients, all with a proven neuropathy. A continuously irregular frequency was found in five patients, three with a proven myopathy and two with undefined pathology. A normal electrical control activity frequency was present in three patients, one with a proven neuropathy and two with undefined pathology. It is suggested that this non-invasive technique may provide a useful screening test of the pathophysiological basis of the functional obstruction in children with chronic idiopathic intestinal pseudoobstruction.
\end{abstract}

(Gut 1992; 33: 1477-1481)

Chronic idiopathic intestinal pseudoobstruction is an uncommon but serious disorder of intestinal neuromuscular function which results in recurrent episodes of intestinal obstruction without a mechanical cause, in vomiting, and in an inability to tolerate oral feeds. Chronic idiopathic intestinal pseudoobstruction is usually caused by disease of the enteric nerves or smooth muscle. This is most often a primary disorder in children but in adults is more commonly secondary to diseases such as diabetes mellitus or scleroderma. Contractile activity of the muscle coats of the gut is regulated by myogenic, neural, and humoral factors. Myogenic control is related to the intrinsic excitability of gastrointestinal smooth muscle cells, and particularly to the constant rhythmic polarisation and depolarisation of the plasmalemmal membranes which gives rise to the electrical slow wave or electrical control activity. Neural and humoral factors modulate these processes to produce patterns of activity appropriate to the circumstances of the gastrointestinal tract at a given time. Abnormalities of electrical control activity have been associ- ated with dysmotility of the gastric antrum, paroxysmally with nausea and vomiting ${ }^{1}$ and persistently with chronic intractable vomiting. ${ }^{2}$

At the present time the diagnosis of chronic idiopathic intestinal pseudoobstruction is based upon invasive investigations, including the demonstration of characteristic patterns of small intestinal motor activity by intraluminal manometry, ${ }^{3+}$ and the histological examination of full thickness intestinal biopsies. As children with diffuse chronic idiopathic intestinal pseudoobstruction frequently vomit, we have investigated gastric electrical control activity as detected by the non-invasive technique of surface electrogastrography in the fasting state in such children to determine if persistently abnormal electrical control activity was present, and if it was related to the underlying pathology of enteric nerves and muscle coats.

\section{Methods}

\section{PATIENTS}

Eleven children with a presumptive diagnosis of chronic idiopathic intestinal pseudoobstruction were investigated. Clinical details of the patients are given in Table I. All had severe symptoms of intestinal pseudoobstruction, including episodic vomiting, abdominal distension, and intolerance of food. None of the patients had evidence of a central nervous system or an autonomic nervous system disorder. The diagnosis was confirmed in all 11 patients by contrast radiography and by antroduodenal manometry performed in a manner previously described. ${ }^{5}$ All had markedly abnormal manometric recordings. In eight of the 11 patients, full thickness intestinal biopsy specimens were available for histological examination. Fifteen control children (age 0.2-17 years, median 11 years) without symptoms of gastrointestinal motor dysfunction formed a control group for surface electrogastrography recordings.

Fasting gastric electrical control activity was recorded and analysed using a modification of

TABLE I Clinical details of the 11 patients with chronic idiopathic intestinal pseudoobstruction

\begin{tabular}{lll}
\hline $\begin{array}{l}\text { Patient } \\
\text { number }\end{array}$ & Age & Symptoms \\
\hline 1 & 3 years & Persistent severe vomiting \\
2 & 9 months & Persistent vomiting and distension \\
3 & 9 months & Persistent vomiting and distension \\
4 & 12 years & Intermittent vomiting \\
5 & 5 years & Intermittent vomiting and distension \\
6 & 16 years & Intermittent vomiting and distension \\
7 & 13 months & Persistent vomiting and distension \\
8 & 5 weeks & Persistent vomiting \\
9 & 6 years & Intermittent vomiting and distension \\
10 & 4 weeks & Persistent vomiting \\
11 & 19 months & Persistent vomiting \\
\hline
\end{tabular}

Correspondence to:

Dr S P Devane, Department of NWB Kin's Hospital, Denmark Hill London SE5 9RS.

Accepted for publication 30 March 1992
Institute of Child Health, Guildford Street, London 


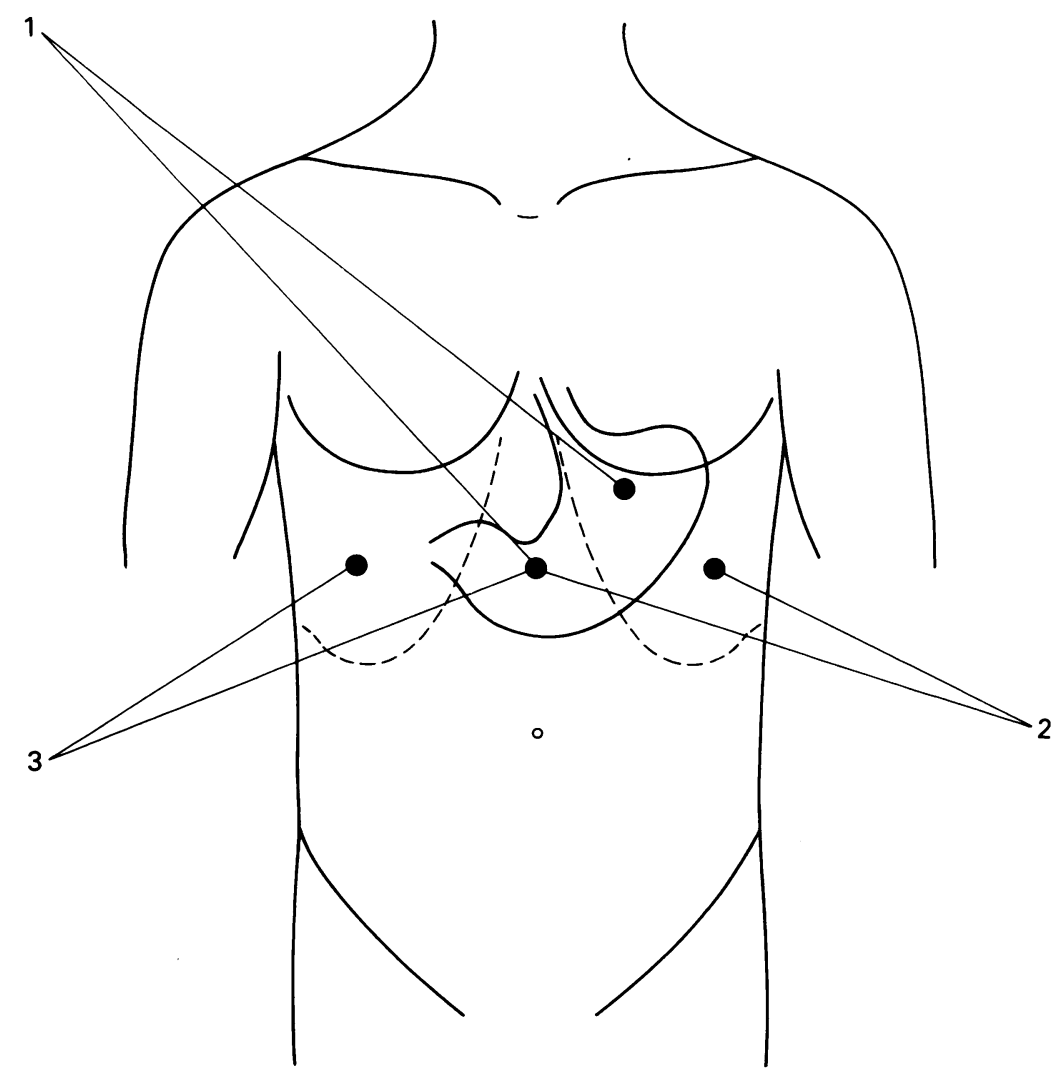

Figure 1: Electrogastrographic electrode placements; the potential difference between the electrodes of pairs 1, 2, and 3 was measured. were obtained in eight of the children. In one patient (patient 4) only routine sections were available for analysis. Biopsy specimens from the remaining seven subjects were divided for routine microscopy (fixation in buffered formalin), for silver staining (fixation in formol saline) and for histochemistry (snap frozen in hexane at $-80^{\circ} \mathrm{C}$ ); a portion was taken for electron microscopy (fixation in $2.5 \%$ glutaraldehyde in $0.1 \mathrm{M}$ cacodylate buffer containing $2 \cdot 5$

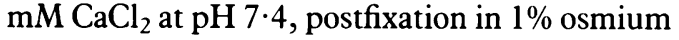
tetroxide and processed into araldite by conventional methods). General morphology was assessed in routine sections. Connective tissue was demonstrated in routine and cryostat sections stained with trichrome and picrosirius methods. Acid phosphatase activity was used to detect lysosomal degradation in smooth muscle, and acetylcholinesterase staining was used to identify nerve fibre distribution in cryostat sections. $^{7}$ The ultrastructure of the intestine was examined in seven patients.

The analysis of the electrogastrogram and the histology was conducted by independent observers in a blind manner.

\section{Results}

\section{ELECTROGASTROGRAPHIC FINDINGS}

The dominant frequencies and the histological abnormalities found in the 11 patients are shown in Table II. A persistently and abnormally fast electrical control activity frequency ( $>5$ cycles/ minute), a tachygastria, was found in three of the subjects with chronic idiopathic intestinal pseudoobstruction and in none of the control subjects. A clear dominant electrical control frequency close to 3 cycles per minute was found in three of the subjects with chronic idiopathic intestinal pseudoobstruction and in all of the control subjects. In five of the subjects with chronic idiopathic intestinal pseudoobstruction, a continuously irregular frequency was present such that no clear dominant frequency could be found in the recording (Fig 2). electrical activity, the signal was digitalised at a frequency of $1 \mathrm{~Hz}$ using an analogue to digital convertor (Data Translation 2801A) and recorded on the hard disk of a desktop personal computer (Zenith Data Systems Z248) for later off line analysis. A running spectral analysis of the real time signal captured on disk was performed. Modular computerised algorithms for data capture, digital conditioning of the captured signal, division into subsections, and frequency analysis (PC-Dats, Prosig Computer Consultants) were used. These modules were ordered and invoked by a Fortran code routine written by the authors (RM-Fortran, Ryan McFarland). Each one hour recording was subdivided into 53 overlapping segments of 256 seconds ( $75 \%$ overlap), each segment was digitally filtered (bandpass Butterworth filter, 0.015-0.25 Hz, slope 24 $\mathrm{dB}$ /octave) and the frequencies present were determined by Fast Fourier Transformation. The peak frequency present in each segment was extracted and the dominant frequency of the one hour recording (that present in the greatest number of segments) was obtained.

Full thickness intestinal biopsy specimens

\section{HISTOPATHOLOGICAL FINDINGS}

In the one subject (patient 4 ) from whom routine sections only of large bowel and ileum were available, histological examination showed an inflammatory process predominantly affecting the myenteric plexus particularly in the

TABLE II Dominant electrical control activity frequency and histological category of the 11 patients with chronic idiopathic intestinal pseudoobstruction

\begin{tabular}{lll}
\hline $\begin{array}{l}\text { Patient } \\
\text { number }\end{array}$ & $\begin{array}{l}\text { Dominant frequency } \\
(\text { cycles/minute })\end{array}$ & Histologv \\
\hline 1 & $6 \cdot 4$ & Neuropathy \\
2 & $8 \cdot 3$ & Neuropathy \\
3 & $9 \cdot 4$ & Neuropathy \\
4 & $2 \cdot 8$ & Neuropathy \\
5 & None & Myopathy \\
6 & None & Myopathy \\
7 & None & Inconclusive examination \\
8 & None & Insufficient material for full \\
9 & None & Noluation histology available \\
& $2 \cdot 2$ & No histology available \\
10 & $3 \cdot 7$ & \\
\hline
\end{tabular}


A

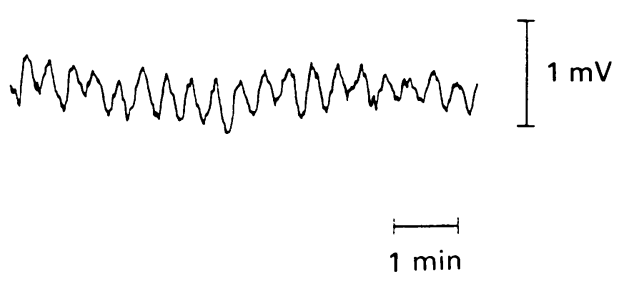

B

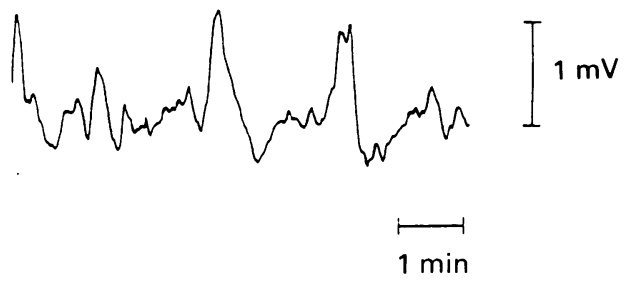

Figure 2: Representations of the recorded electrical control activity from (a) a subject with a normal frequency of electrical control activity; $(b)$ a subject with a continuously irregular electrical control activity frequency; (c) $a$ subject with a tachygastria. In each case, a short extract from the original recording, and a pseudo-3-dimensional representation of the frequency distribution in each of the 256 second segments obtained for analysis from a one hour recording is shown.

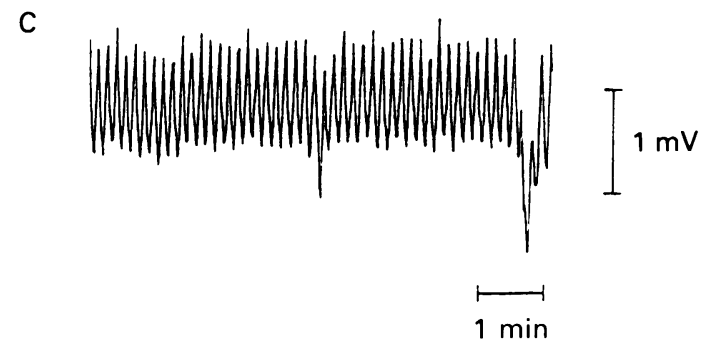

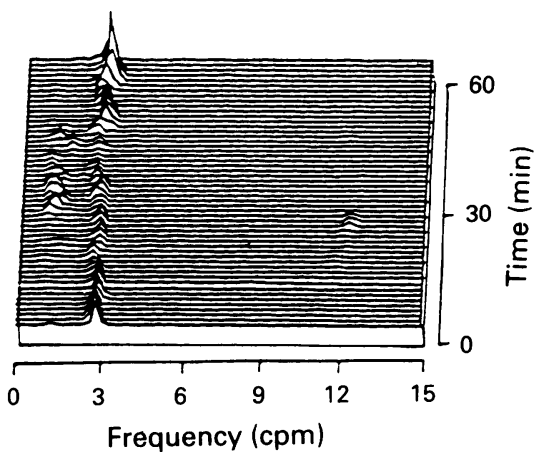
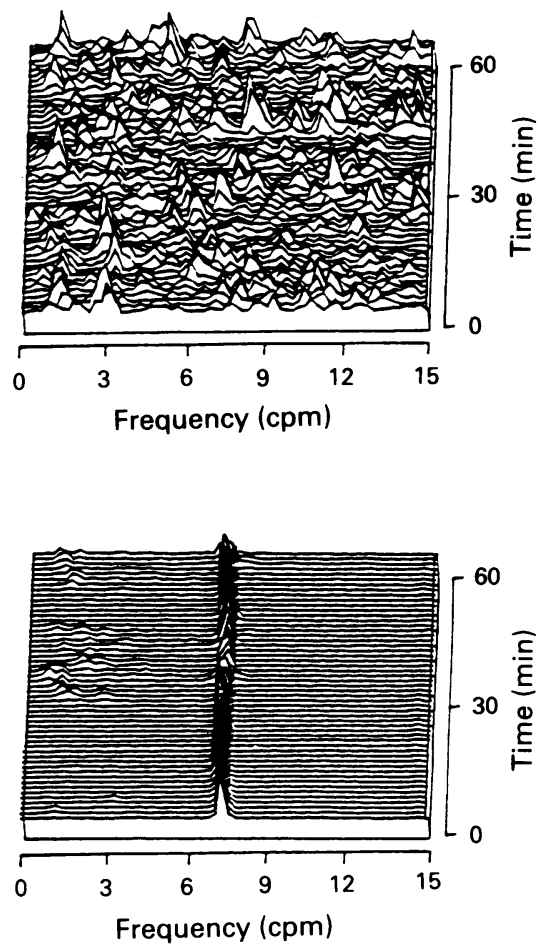

appendix but extending to the small intestine. Neurones in the myenteric plexus were not identified in the colon and were sparse and appeared degenerate in the appendix and ileum.

Pathological changes either in the neural plexus or in the smooth muscle coats were not evident in routine or cryostat sections, or on histochemical staining, in the seven subjects from whom full thickness bowel samples were available for further analysis. In three of these patients (patients 5, 6, and 7), ultrastructural examination, however, showed abnormalities of smooth muscle fibres whilst normal argyrophilic neurones were seen in the silver preparations. In one patient (patient 8) there was no conclusive ultrastructural evidence of a myopathic change, and the neurones appeared ultrastructurally normal. No material was available for silver staining from this patient. In the remaining three subjects (patients 1, 2, and 3) silver staining revealed a neuropathic abnormality with absent or severely depleted and ill defined argyrophilic neurones in the myenteric plexus. Electron microscopy of the bowel from one of these patients (patient 1) also showed atrophic and shrunken neurones in the myenteric plexus. Intestinal smooth muscle from these patients was ultrastructurally unremarkable.

\section{RELATIONSHIP BETWEEN}

ELECTROGASTROGRAPHIC, HISTOPATHOLOGICAL, AND MANOMETRIC FINDINGS

The four patients with proven neuropathy had severely disturbed propagation and organisation of antroduodenal motor activity. All three patients with proven myopathy had abnormal conformation of phase III of the migrating motor complex with a low amplitude of contraction. Two of the four patients without a proven histological diagnosis had low amplitude poorly formed phase III complexes. Of the remaining two patients, one had both low amplitude of contractions and severe disorganisation of activity, and one (patient 8 ) has no motor activity detected.

All three subjects with a tachygastria (patients 1,2 , and 3) had a proven neuropathy of the myenteric plexus whereas three of the five patients with a continuously irregular frequency of the electrical control activity had clear evidence of a myopathy of the muscle coats on ultrastructural studies. In the remaining two patients the ultrastructural changes in the muscle coats were inconclusive in one case (patient 8) and the histological material was inadequate for diagnosis in the other case (patient 9). 


\section{Discussion}

Chronic idiopathic intestinal pseudoobstruction is a disorder of motor function of the gastrointestinal tract as a result of disease of enteric nerves or smooth muscle cells. We and others ${ }^{8-10}$ have described the histological characteristics of full thickness intestinal biopsies in patients with visceral myopathy and neuropathy. A variety of abnormalities including inflammatory cell infiltration and Schwann cell proliferation, hypoganglionosis, and hyperganglionosis have been described in those with disease of the enteric nervous system. In muscle disease degeneration of muscle fibres and replacement with fibrous tissue have been seen. In our patients, four had evidence of a neuropathy (one with associated inflammatory changes) and three had evidence of a myopathy.

Manometric and electrophysiological studies of intestinal myoelectrical and motor activity in patients with pseudoobstruction have shown clearly different patterns of activity in those with disorders of the enteric nervous system compared with those with muscle disease. ${ }^{3+811}$ In the former, disturbed conformation and propagation of phase III of the migrating motor complex together with bizarre waveforms of normal or increased amplitude are usually seen, whereas in the latter very low amplitude or absent contractions are the rule. In our patients, those with proven neural and muscular pathology conformed to these patterns of motor activity. In addition, two others without a histologically proven diagnosis had a pattern suggestive of a myopathic process.

Smooth muscle cells of the gastric body and antrum exhibit inherent rhythmic polarisation and depolarisation of the electrical potential across the cell membrane. The frequency of this rhythmic activity is determined by a group of cells on the greater curvature which have been called the gastric pacemaker. This slow wave or electrical control activity is entrained throughout the stomach because of electrical coupling between adjacent muscle cells and a differential in excitability of the muscle cells between the gastric corpus and the antrum. ${ }^{12}$ In the human antrum, the frequency of the electrical control activity is close of $0.05 \mathrm{~Hz}$ (3 cycles/minute). This electrical control activity can be detected by electrodes placed surgically in the bowel wall ${ }^{13}$ or by electrodes placed against the mucosal surface by intubation techniques. The former has provided useful information in cases requiring laparotomy. ${ }^{14}$ The latter suffers from unstable mucosal contact, although the use of improved fixation techniques such as magnetic stabilisation has reduced this problem. ${ }^{15}$ The invasive nature of intubation techniques, however, restricts its usefulness in pediatric practice. Recordings of gastric electrical control activity made from surface electrodes have been shown to correlate well with mucosal recordings ${ }^{16}$ and this together with improvements in detection methods and their wider availability has led to increased use of surface electrogastrography in the investigation of gastrointestinal symptoms. The advent of powerful desktop computers and the introduction of the technique of running spectal analysis by Van der Schee ${ }^{6}$ has allowed objective analysis of the recorded signals, superceding the use of visual analysis techniques."

Abnormality of the electrical control activity has been shown to occur in association with motion sickness ${ }^{18}$ in patients with idiopathic gastroparesis, ${ }^{19}$ unexplained nausea and vomiting, 'and anorexia nervosa, ${ }^{17}$ and in response to vagotomy $^{20}$ and to administration of glucagon. ${ }^{15}$ One case report has been published describing a gastric electrical control activity close to $0.05 \mathrm{~Hz}$ (3 cycles/minute) with normal histology in a child with an idiopathic motility disorder. ${ }^{1+}$ Tachygastria has been reported in a child with gastric atony, ${ }^{21}$ who later developed the full clinical picture of CIIP. ${ }^{22}$ To our knowledge, this is the first report of persistent antral dysrhythmias in a series of patients with chronic idiopathic intestinal pseudoobstruction and known disease of enteric nerves or smooth muscle.

Three of the four patients with a known neuropathy but none of the control group and none of the patients with a known myopathy had a persistent tachygastria in the fasting state. Thus detection of a persistent tachygastria in the fasting state is highly suggestive of a neuropathic basis for the pseudoobstruction. The mechanism whereby a neuropathy may produce a tachygastria is uncertain. The neural abnormalities that could induce a tachygastria include loss of intrinsic inhibitory innervation or lack of extrinsic autonomic inhibition. We have no evidence in any of our patients, after investigation of the central and autonomic nervous systems, that extrinsic neural control was disordered. It is clear, however, that a variety of conditions affecting the extrinsic nerves and the central nervous system may result in tachygastria, ranging from children with cerebral palsy, ${ }^{23}$ to motion sickness, ${ }^{18}$ anorexia nervosa, ${ }^{17}$ and vagotomy. ${ }^{24}$ In these conditions, with the exception of cerebral palsy, the tachygastria is usually paroxysmal, and the increased frequency is somewhat variable. We were impressed that the tachygastria in our patients with neuropathic pseudoobstruction was both persistent and raised consistently in the fasting state. We were unable to explore systematically the electrical control activity in the postprandial state because of the patients' intolerance to food. We would suggest that in neuropathic pseudoobstruction it is more likely that the tachygastria is induced by loss of intrinsic inhibitory innervation which may normally modify the frequency of polarisation of the plasmalemmal membrane of the gastric smooth muscle cells.

The presence of a continuously irregular frequency such that no dominant frequency could be shown was found in the three patients with a proven myopathy and in two patients with a manometric pattern suggestive of muscle disease. Electrical control activity is caused by the entrainment of the fluctuations of the transmembrane potentials of individual smooth muscle cells. The apparent continuously irregular frequency recorded, which contains high and low frequency components, may have several explanations. It may be the result of inability to maintain a constant frequency of electrical control activity and this may be due to 
the effects of muscle disease on groups of muscle cells producing populations with altered frequencies. Alternatively, it is possible that the abnormal recordings are the result of poor summation of the electrical signal in the abnormal muscle syncytium and poor projection onto the body surface. A reduced amplitude of the electrical control activity may result in a marked diminution of the signal to noise ratio making it difficult to extract the true dominant frequency. In addition, it may be considered that the frequency pattern results from a technically unsatisfactory recording. We think the last explanation is unlikely as unsatisfactory recordings produced on our equipment result in a signal with an overwhelming predominance of low frequencies of $<0.025 \mathrm{~Hz}$ (unpublished observations). At the present time, it is not possible to distinguish between the other alternative explanations, though it may be that both factors operate to modulate the pattern of frequencies recorded at the body surface. In the future, computerised spatial mapping of the distribution of frequencies in the manner now being used for electroencephalographic mapping of epileptic foci may provide a non-invasive alternative to multiple implanted electrodes to help distinguish between the above explanations.

In conclusion we have found a persistent tachygastria in the fasting state in three of four patients with chronic idiopathic intestinal pseudoobstruction with a known neuropathic basis for their condition, and in none of 15 control patients, none of three patients with chronic idiopathic intestinal pseudoobstruction as a result of muscle disease, and none of four with chronic idiopathic intestinal pseudoobstruction with an unknown basis. In the three patients with muscle disease there was absence of a dominant frequency. We suggest that surface electrogastrography may offer a non-invasive means of screening patients with suspected neuromuscular disease of the gut causing persistent vomiting and episodes of functional obstruction.

SD would like to acknowledge generous support from the Janssen Research Foundation. This work was published in part in abstract form following presentation at the International Symposium on Gastrointestinal Motility, Vienna, September 1989.

1 Geldof H, Van der Schee EJ, van Blankenstein M, Grashuis JL. Electrogastrographic study of gastric myoelectric activity in patients with unexplained nausea and vomiting Gut 1986; 27: 799-808.

2 Cucchiara S, Janssens J, Vantrappen G, Geboes K, Ceccatelli $P$. Gastric electrical dysrhythmias (tachygastria and tachyarrhythmia) in a girl with chronic intractable vomiting. F Pediatr 1986; 108: 264-7.

3 Stanghellini V, Camilleri M, Malagelada J-R. Chronic idiopathic intestinal pseudo-obstruction: clinical and intestinal manometric findings. Gut 1987; 28: 5-12.

4 Hyman PE, McDiarmid SU, Napolitano J, Abrams CE, Tomomasa T. Antroduodenal manometry in children with chronic intestinal pseudo-obstruction. $\mathcal{F}$ Pediatr 1988; 112:

5 Fenton T, Harries JT, Milla PJ. Disordered small intestinal motility: a rational basis for toddler's diarrhoea. Gut 1983

24: 897-903.
6 Van der Schee EJ, Grashius JL. Running spectrum analysis as an aid in the representation and interpretation of electrogastrographic signals. Med Biol Eng Comput 1987; 25 $57-62$.

7 Filipe IM, Lake BD, eds. Histochemistry in pathology. 2nd ed Edinburgh: Churchill Livingstone, 1990: 444-63.

8 Milla PJ, Lake BD, Spitz L, Nixon HH, Harries JT, Fenton TR. Chronic idiopathic intestinal pseudo-obstruction in infancy: a smooth muscle disease. In: Labó $G$, Bortolotti $M$ eds. Gastrointestinal motility. Verona: Cortina International, 1983: 125-31.

9 Krishnamurthy S, Schuffler MD. Pathology of neuromuscular disorders of the small intestine and colon. Gastroenterology disorders of the sma
1987; 93: 610-39.

10 Navarro J, Sonsino E, Boige N, Nabarra B, Ferkadji L, Mashako LMN, et al. Visceral neuropathies responsible for chronic intestinal pseudo-obstruction syndrome in pediatric practice: analysis of 26 cases. $\mathcal{F}$ Pediatr Gastroenterol Nutr 1990; 11: 179-95.

11 Wozniak E, Fenton TR, Milla PJ. Fasting small intestinal motor activity in chronic idiopathic intestinal pseudoobstruction. Pediatr Res 1984; 18: 1060

12 Kelly KA. Differential responses of the canine gastric corpus and antrum to electrical stimulation. Am $\mathcal{J}$ Physiol 1974 226: $230-4$.

13 Alvarez WC, Mahoney LJ. Action current in stomach and intestine. Am $\mathcal{F}$ Physiol 1922; 58: 476-93.

14 Bland EL, Karaus M, Glicklis M, Sarna SK, Werlin SL. Gastrointestinal myoelectric activity in an infant with Gastrointestinal myoelectric activity in an infant with congenita

15 Abell TL, Malagelada J-R. Glucagon-evoked gastric dysrhythmias in humans shown by an improved electrogastrographic technique. Gastroenterology 1985; 88 1932-40.

16 Hamilton JW, Bellahsene BE, Reichelderfer M, Webster JG Bass P. Human electrogastrograms, comparison of surface and mucosal recordings. Dig Dis Sci 1986; 31: 33-9.

17 Abell TL, Malagelada J-R, Lucas AR, Brown ML, Camilleri M, Go VLW, et al. Gastric electromechanical and neurohormonal function in anorexia nervosa. Gastroenterology 1987; 93: 958-65.

18 Koch KL, Stern RM, Vasey MJ, Seaton JF, Demer LM Harrison TH. Gastric myoelectric and endogenous neuroHarrison TH. Gastric myoelectric and endogenous neuroendocrine responses to ill
enterology 1988; 95: 875.

19 Bortolotti M, Pinotti R, Barbara L. Gastric myoelectric activity in patients with chronic idiopathic gastroparesis. Gastroenterology 1989; 95: 857

20 Nelsen TS, Kohatsu S. Clinical electrogastrography and its relationship to gastric surgery. Am $\mathcal{F}$ Surg 1968;116: 215-22.

21 Telander RL, Morgan KG, Kreulen DL, Schmalz PF, Kelly KA, Szurszewski JH. Human gastric atony with tachygastria and gastric retention. Gastroenterology 1978; 75: 497 501 .

22 Malagelada JR. Manometric diagnosis of gastrointestinal motility disorders. New York: Thieme Medical, 1986: 109.

23 Devane SP, Bisset WM, Milla PJ. Persistent tachygastria in severe nausea and vomiting. Pediatr Res 1989; 26: 275.

24 Geldof $H$, Van der Schee EJ, Van Blankenstein $M$, Smout AJPM, Akkermans LMA. Effects of highly selective vagotomy on gastric myoelectric activity, an electrogastrographic study. Dig Dis Sci 1990; 35: 969-75. 